\title{
Editorial
}

\section{Frank Ettrich}

Die ,Verheftung“ von Nummer 3/2019 des Berliner Journals für Soziologie erfolgt zu einem Zeitpunkt, zu dem die „Lockerungen“ des politisch verfügten „Lockdowns“ im Angesicht der Gefahren und Risiken von Covid-19 es uns wieder gestatten, mehr als das digital Mögliche in Angriff zu nehmen. Auch für die Macher des BJS galt fast zehn Wochen lang, was Michel Foucault für das 17. Jahrhundert als soziale Konsequenzen des Quarantänereglements, ,wenn sich die Pest in einer Stadt ankündigte“, folgendermaßen beschreibt: „Der Raum erstarrt zu einem Netz von undurchlässigen Zellen. Jeder ist an seinen Platz gebunden. Wer sich rührt, riskiert sein Leben: Ansteckung oder Bestrafung.“ (Foucault 1977, S. 251) Die digitale Durchlässigkeit der „Zellen“ in der Gegenwart kompensiert keinesfalls vollständig das Einfrieren jener Netzwerke und institutionellen Routinen, die die mobilen und flexiblen Lebens- und Arbeitsweisen gerade im wissenschaftlichen Bereich für gewöhnlich ermöglichen.

Die Welt im buchstäblichen Sinne des Wortes steht noch immer „im Banne“ von Covid-19, auch wenn sich das Epizentrum des Infektionsgeschehens fürs Erste von Europa auf die beiden Amerikas und den indischen Subkontinent verlagert hat. In den Monaten März und April 2020 befanden sich fast 60\% der Menschheit im Lockdown, dem Zustand eingeschränkter Bürgerrechte und der weitgehenden Stilllegung des öffentlichen Lebens. Die in ihrer agency-Fähigkeit seit langem infrage gestellten europäischen Nationalstaaten (und auch die EU) sehen sich und ihre Gesundheitssysteme inzwischen wieder in der Lage, Versuche einer tastenden Normalisierung von Politik, Wirtschaft und Gesellschaft zu unternehmen. Wie die Normalität der aus diesen Normalisierungsversuchen hervorgehenden Gesellschaften aussehen wird, dürfte auch für die Sozialwissenschaften in nächster Zukunft ein drängendes Thema sein.

F. Ettrich $(\bowtie)$

Erfurt, Deutschland

E-Mail: frank.ettrich@uni-erfurt.de 
Es macht keinen Sinn, krampfhaft einen Bogen von der Corona-Pandemie zum Inhalt eines Heftes spannen zu wollen, das zum überwiegenden Teil vor dem 12. März 2020 - dem Tag, an dem die WHO das globale Infektionsgeschehen zu einer Pandemie erklärte - entstanden ist. Vielleicht ist für die nunmehr einsetzende sozialwissenschaftliche Diskussion und Forschung über die gesellschaftlichen Konsequenzen der Corona-Krise aber darauf zu verweisen, dass das Neue an der gegenwärtigen Situation vor allem darin besteht, dass erstmals wieder seit der ,Spanischen Grippe“ 1918-1920, der mit Schätzungen von 20 bis 100 Mio. Toten pandemischen „Urkatastrophe“ des 20. Jahrhunderts, auch die Kernländer der westlichen Welt mit den Gefahren einer Pandemie konfrontiert sind. Andernorts ist man mit diesen Gefahren, wie sie - von der siebten Cholera-Pandemie über „Hongkong-“, „Vogel-“ und „Schweinegrippe“ bis hin zum SARS-Ausbruch - eine sich formierende und akzelerierende Weltgesellschaft immer schon begleiten, vertrauter geblieben. Doch wohl keine Krise der an Krisen nicht armen jüngeren und jüngsten Geschichte hat die Welt so durcheinandergebracht wie die gegenwärtige Corona-Krise. Dabei kann die Spezifik des Viruses allein die Wucht des Einschlags kaum erklären - auch wenn SARS-CoV-2 ein besonders tödlicher und ansteckender Vertreter der Klasse der Coronaviren ist. Die Ursachen der unikalen Weise, in der es unser gesellschaftliches Zusammenleben beeinflusst und beeinträchtigt, müssen vielmehr in der Art und Weise gesucht werden, in der sein Wirt, der Mensch, gegenwärtig sein Zusammenleben gestaltet und organisiert. Die Weltgesellschaft ist eine Weltrisikogesellschaft, und die Risikogesellschaft, das betonte Ulrich Beck schon 1986 in seinem zeitdiagnostischen Eröffnungsstatement zur neuen Moderne, ist „eine Katastrophengesellschaft. In ihr droht der Ausnahmezustand zum Normalzustand zu werden" (Beck 2003, S. 105). Aber schon Beck neigte dazu, virale Pandemien in seinen Listen der zivilisatorisch gemachten Plagen zu ignorieren. Wäre die Korrektur dieses blinden Fleckes ein möglicher Ausgangspunkt der sozialwissenschaftlichen Ver- und Bearbeitung unserer gegenwärtigen kollektiven Krisenerfahrungen? Sollten wir Pandemien wie die, die die Welt gerade erlebt, ebenso wie die Risiken der „Großtechnik“ in das Register der „normalen Katastrophen“ (Charles Perrow) aufnehmen?

Der Lockdown war die Zeit der medialen Hegemonie von Virologen und Epidemiologen, deren datengestützte Kakophonie auch für Natur- und Gesundheitswissenschaftler deutlich machte, dass die Mechanismen der Mediengesellschaft eine hochriskante emergente Dimension wissenschaftlichen Tuns sind. Auch einzelne Vertreterinnen und Vertreter sozialwissenschaftlicher Disziplinen übten sich im „Risiko der Zeitdiagnose“. Für Anhänger der makrosoziologischen Differenzierungstheorie stellt der radikale Lockdown - wie Rudolf Stichweh (2020) formulierte - eine zeitweilige „Simplifikation des Sozialen“ dar: Das zum „Krankheitssystem“ mutierte Gesundheitssystem wird zum „Ganzen der Gesellschaft“, und zwar das Krankheitssystem ,in seiner extremsten Form als totale Institution, also als Intensivmedizin, die das Individuum in der Gesamtheit seiner Lebensvollzüge steuert und in dieser Form der Letztbedeutsamkeit des Individuums Rechnung trägt“. „Normalisierung“, die in dieser Perspektive den "Neustart“ der gesellschaftlichen Funktionssysteme bedeutet, werde ohne „Strukturbrüche“ nicht zu haben sein. Für die unmittelbare Zeit der Lockerungen lässt sich zunächst beobachten, dass die Kontrolle des In- 
fektionsgeschehens in der Bundesrepublik flächendeckend den Gesundheitsämtern übereignet wurde.

Gewissermaßen „sozialontologisch“ den Gegenpol zur differenzierungstheoretischen Perspektive bildet Tilman Allerts (2020) Ruf nach einer „Soziologie der Ungeselligkeit“, einer „Choreographie anomischer Vorgänge“, die die aus dem Trott oder Tritt geratene Sozialordnung jenseits von Mustern wie Apathie, Panik oder Flucht wahrzunehmen gestattet. Fast schon optimistisch stimmt die Deutung von „social distancing" und Maskenpflicht aus zivilisationstheoretischer Perspektive. Reinhard Blomert (2020) hält es für denkbar, dass der gegenwärtige Immunisierungsstress zu einem Zivilisierungsschub im Sinne der Figurationssoziologie von Norbert Elias führt: ,Eine Gesellschaft, die sich in eine Immunstation verwandelt hat, wird sich so einfach nicht wieder in den früheren Stand versetzen lassen. Die Ubiquität der Maßnahmen im Kampf gegen Covid-19 zeigt das neue Gewicht an, das die Gesundheit erhalten hat - und damit auch der aus der Gefahrenzone herausgezogene, mit zivilisatorischer Kunstfertigkeit isolierte Körper. Dass damit zugleich eine Entfremdung einhergeht, ist die Ambivalenz, die diesen ganzen historischen Prozess begleitet.“ Die kontakt- und berührungslose Gesellschaft als eine Folge von Covid-19 also.

Diese wenigen Beispiele aus einer ganzen Reihe sozialwissenschaftlicher und soziologischer Situationsbeschreibungen der gegenwärtigen Krisenlage sollen an dieser Stelle genügen, um anzudeuten, dass die Sozialwissenschaften nicht in eine Angst- und Duldungsstarre verfallen sind. Die vorliegenden zeitdiagnostischen Texte lassen sich übergreifend als Versuche verstehen, die virale Krisensituation im Licht der Fortschreibung etablierter paradigmatischer Orientierungen und Denkmuster zu sehen. Lediglich im Globalisierungsdiskurs häufen sich die Stimmen, die die Covid-Pandemie als mögliche Zäsur und Aufforderung zur Restrukturierung der beschleunigten, neoliberalen Globalisierungsprozesse der vergangenen 30 Jahre zu thematisieren versuchen. Als ,finale Entzauberung der Globalisierung“ hat schon früh Ulrich Menzel (2020) den „Corona-Schock“" gesehen, wobei er bei seiner Suche nach historischen Parallelen bis ins Jahr 1320 zurückgegangen ist, in dem der Ausbruch der Pest in der zentralchinesischen Provinz Hubei das Ende einer welthistorischen Globalisierungsepoche einleitete. Baute sich damals das pandemische Geschehen über nahezu ein halbes Jahrhundert auf, sind es heute Wochen und Monate. Finale Entzauberung heißt hier wohl, dass die Corona-Krise die Kosten transparent gemacht hat, die mit der unablässigen Ausweitung von internationaler Arbeitsteilung und Welthandel verbunden sind - Kosten, zu denen neben Klimawandel und Migration auch die Verbreitung von Viren zu rechnen ist.

Allerdings bedeutet Globalisierung heute, wie Paul Virilio bereits vor mehr als einer Generation betont hat, vor allem Digitalisierung. $\mathrm{Zu}$ den bisherigen Gewinnern des Lockdowns realwirtschaftlicher Prozesse gehören zweifellos die großen Player der Digitalwirtschaft, also v. a. Google, Amazon, Microsoft, Apple und Facebook, deren Börsennotierungen selbst den Lockdown weitgehend unbeschadet (oder im Fall von Amazon mit erheblichen Gewinnen) überstanden haben. Ein Newcomer wie Zoom erlebte in wenigen Wochen geradezu einen kometenhaften Aufstieg seines Geschäftsmodells. Dass die sanitäre Krisenkonstellation der letzten Monate die Digitalisierung aller gesellschaftlichen Lebens- und Kommunikationsvollzüge 
nachhaltig beschleunigt hat, gehört zu den wenigen gesicherten Wahrheiten dieser Zeit.

Der Analyse der großen Internetunternehmen als den „strukturbildenden, regelsetzenden und handlungskoordinierenden Kernakteuren" des Internets, die Ulrich Dolata in seinem Beitrag zum vorliegenden Heft unterbreitet, kommt damit dann doch eine noch größere Aktualität zu, als sie vor der Coronakrise ohnehin schon hatte. Mit dem Betrieb von globalen Plattformen wächst den großen Internetfirmen eine technische, ökonomische und soziale Regelungshoheit im Netz zu, die den Autor von der Übernahme ,quasi-hoheitlicher“, d.h. originär staatlicher Aufgaben durch privatwirtschaftliche Akteure sprechen lässt. Dolata analysiert die intentionale Strukturbildung durch plattformbetreibende Internetunternehmen für zwei große Regelungsbereiche: die privatwirtschaftliche Organisierung und Regelung unternehmenseigener Märkte für Dienstleistungen, Arbeit und Produkte sowie die Gestaltung von sozialen Handlungsrahmen durch die „Kuratierung“ von Inhalten, Kommunikation und Öffentlichkeit. Vor allem in diesen beiden Bereichen der Regulierung durch Plattformen sieht der Autor das „Neue und Disruptive“ des Geschäftsmodells der privatwirtschaftlich betriebenen Internetplattform. Als Ausblick seiner Argumentation diskutiert Dolata die zivilgesellschaftlichen und politischen Ansatzpunkte und Grenzen der Regulierung von Plattformen. Die bisherigen gesetzgeberischen und politischen Ergebnisse bei dieser Frage sieht der Autor eher skeptisch.

„Internetpolitik“ ist inzwischen ein eigenständiges Politikfeld, für das immer wieder auch die Forderung nach einem eigenen Ministerium zu vernehmen ist. Wie entstehen eigentlich neue Politikfelder? Maximilian Hösl und Ronja Kniep rekonstruieren in ihrem Aufsatz aus einer feldtheoretischen Perspektive den Entstehungsprozess des vergleichsweise neuen Politikfeldes „Internetpolitik“, wobei sie sich auf die organisationale Ausdifferenzierung von Kompetenzen, Organisationsstrukturen und Ressourcen auf der Politikebene von Bundesministerien (Bundesministerium für Wirtschaft, Bundesministerium des Innern und Bundesministerium für Verkehr und digitale Infrastruktur) der Bundesrepublik Deutschland im Zeitraum von 1995 und 2014 konzentrieren. Neben der leitenden Forschungsfrage nach der Entstehung von Politikfeldern auf nationaler Ebene sind Hösl und Kniep vor allem daran interessiert zu eruieren, inwiefern sich politische Institutionalisierungsprozesse mit den Mitteln des diskursiven Institutionalismus angemessen und tiefer analysieren lassen. Die diskursive und konfligierende Identifikation ,gemeinwohlorientierter Schutzgüter", hier des Internets, bildet nach Ansicht der Autor*innen den Ausgangspunkt komplexer Formierungsprozesse von politischen (Meta-)Feldern, in denen Diskurse und organisationale Reorganisationsprozesse zusammenwirken.

Ein möglicherweise unspektakuläres, symbolisch aber markantes Element des Übergangs zum ,aktivierenden“ Sozialstaat in der Bundesrepublik Deutschland war die Reform des Erwerbsminderungsrechts im Jahr 2001, die mit einer Befristungsregelung für den Bezug von Erwerbsminderungsrenten das Primat vom Sozialleistungsbezug auf den Wiedereintritt in Erwerbstätigkeit umstellte. Martin Brussig, Susanne Eva Drescher und Thorsten Kalina werten diese Reform in ihrem Beitrag als die ,einschneidendste Veränderung seit der Einführung der Rentenversicherung 1889 bzw. seit Einführung der Versicherung gegen Berufsunfähigkeit 1913“. Auf der Basis des Scientific Use File der Versichertenkontenstichprobe der Deutschen Ren- 
tenversicherung aus dem Jahr 2015 und damit für knapp 1,9 Mio. Rentenversicherte, die in ihrem Erwerbsleben Phasen der Erwerbsminderung durchleben, untersuchen die Autor*innen, wie sich die Erwerbschancen - differenziert nach personalen und erwerbsbiografischen Merkmalen - nach dem Ende eines Erwerbsminderungsrentenbezugs bisher darstellten. Sozialpolitische Empfehlungen zur Feinjustierung und Weiterentwicklung der Erwerbsminderungsrente runden den Beitrag von Brussig, Drescher und Kalina ab.

In ihrer polemischen Jugendschrift Die heilige Familie aus dem Jahr 1845 formulierten der damals 24-jährige Friedrich Engels und der 26-jährige Karl Marx in der apodiktischen Sprache der Junghegelianer, von denen sie sich mit dieser Schrift zu emanzipieren suchten: „Die besitzende Klasse und die Klasse des Proletariats stellen dieselbe menschliche Selbstentfremdung dar. Aber die erste Klasse fühlt sich in dieser Selbstentfremdung wohl und bestätigt, weiß die Entfremdung als ihre eigne Macht und besitzt in ihr den Schein einer menschlichen Existenz; die zweite fühlt sich in der Entfremdung vernichtet, erblickt in ihr ihre Ohnmacht und die Wirklichkeit einer unmenschlichen Existenz." (Marx und Engels 1962, S. 37) Seit es arbeitswissenschaftliche und -soziologische Forschungen gibt, ist das, was Marx und Engels hier als „Ohnmacht" und ,vernichtende“ Entfremdung aufrufen, ein zentrales Thema der Arbeits- und Berufsforschung. Mit der verstärkten Einführung marktzentrierter Kontrollmodi auch in den Tätigkeitsfeldern hochqualifizierter, bisher vergleichsweise autonomer professioneller Berufe nehmen Befunde über Gefühle der „,Ohnmacht“ und „Entfremdung“ auch in diesen Sektoren der Erwerbsarbeit zu. Auf der empirischen Grundlage von 40 Interviews mit Ärzt*innen und Sozialarbeiter*innen in Führungspositionen gehen Friedericke Hardering und Mascha Will-Zocholl in ihrer Abhandlung der Frage nach, wie sich in Feldern professioneller Dienstleistungsarbeit heute die Aneignungsweisen von Arbeitsinhalten und Arbeitssituationen als aktive Auseinandersetzungsprozesse zwischen den Polen „Sinnbewahrung“ und „Sinngestaltung“ vollziehen. Die Autorinnen destillieren aus ihrem Interviewmaterial drei Aneignungsweisen von Arbeit heraus, die eine informative Differenzierung der aktiv-gestaltenden Verarbeitungsformen und -mechanismen von Entfremdungserfahrungen durch die Beschäftigten in der gegenwärtigen Berufswelt hochqualifizierter Dienstleistungsarbeit erlauben.

Mit dem Wandel der Bundesrepublik Deutschland zu einer Einwanderungsgesellschaft haben nahezu flächendeckend Einbürgerungsfeiern in den Integrationsalltag deutscher Städte und Gemeinden Einzug gehalten. In einer rekonstruktiven ethnografischen Analyse von 14 solcher Einbürgerungsfeiern untersucht Maria Jakob in ihrem Aufsatz diese vergleichsweise neue Form der politisch-rituellen Repräsentation von Staatsbürgerschaft und Zugehörigkeit in der deutschen Gesellschaft. Die Autorin stellt dabei nicht den ,rites de passage“-Aspekt solcher Feiern in den Mittelpunkt ihrer Untersuchung, vielmehr interessiert Jakob der Gesichtspunkt der symbolischen Arbeit, die seitens offizieller Vertreter*innen, aber durchaus auch der Neubürger*innen bei solchen außeralltäglichen Anlässen geleistet wird, um Zugehörigkeit anders als im Sinne von Homogenität und nationaler Identität zu kommunizieren und zu begründen. Bei aller Ambivalenz des Geschehens gelingt dies in den meisten der analysierten Fälle. Es sind vor allem vier symbolische Codes, die im Zentrum der analysierten Praktiken stehen: „das Lokale“, „(Hoch-)Kultur“, 
„Leistung“ und „Bürgerlichkeit“. Im Prozess des Zusammenwirkens dieser Einbürgerungsmarker und ihrer rituellen Einbettung sieht die Autorin eine allmähliche symbolische Dekonstruktion nationaler Zugehörigkeit am Werke.

Am 14. Juni 2020 jährt sich der Todestag von Max Weber zum 100. Mal. Christian Martys Essay zum Motiv der Vornehmheit bei Weber, ein Resultat der Auseinandersetzung Webers mit Friedrich Nietzsche und Georg Simmel, lässt deutlich werden, dass Vertreter*innen einer neuen Wissenschaftler*innen-Generation dem Werk Max Webers auch nach dem institutionellen Abgang der Forschergeneration, die die Weber-Renaissance des 20. Jahrhunderts geprägt hat, weiterhin interessante Forschungsfragen abzugewinnen vermögen.

In seinem Review-Essay bespricht Walther Müller-Jentsch zehn der zwischen 2016 und 2019 zur Kritischen Theorie erschienenen Publikationen. Der Autor sieht vor allem in der anhaltenden Rezeption der „Kulturindustrie“-These die wichtigste Barriere einer bloßen Historisierung der ersten und zweiten Generation der Kritischen Theorie. Einen anhaltenden und eher zunehmenden Stachel der aktuellen Diskussion zum Thema macht Müller-Jentsch in der Verortung von Jürgen Habermas in der Geschichte der Kritischen Theorie aus.

Den Abschluss des vorliegenden Heftes bilden - wie leider so häufig - zwei professionspolitische Texte, die ihre Entstehung traurigen Anlässen verdanken. Stefan Schmalz würdigt in seinem Nachruf Immanuel Wallerstein, der am 31. August 2019 verstorben ist, als Begründer und Galionsfigur der sozialwissenschaftlichen Weltsystemanalyse. Am 23. Januar 2019 verstarb Erik Olin Wright, der bedeutende Innovator und Vertreter einer marxistischen Klassenanalyse in der internationalen Soziologie. Den Nachruf auf Wright schrieb Hans-Peter Müller.

\section{Literatur}

Allert, T. (2020). Soziologie der Ungeselligkeit: Wappnet euch mit Gleichmut. Frankfurter Allgemeine Zeitung v. 27.03.2020, https://www.faz.net/aktuell/wissen/geist-soziales/gedanken-zu-einer-soziologiedes-geselligkeitsverzichts-16682315.html. Zugegriffen: Juni 2020.

Beck, U. (2003) [1986]. Risikogesellschaft. Auf dem Weg in eine andere Moderne. Frankfurt a. M.: Suhrkamp.

Blomert, R. (2020). Gesellschaft im Hygienestress: Händewaschen nie vergessen! Frankfurter Allgemeine Zeitung v. 26.04.2020, https://www.faz.net/aktuell/wissen/geist-soziales/haendewaschennie-vergessen-die-gesellschaft-ist-im-immunisierungsstress-16723879.html?premium. Zugegriffen: Juni 2020.

Foucault, M. (1977). Überwachen und Strafen. Die Geburt des Gefängnisses. Frankfurt a. M.: Suhrkamp.

Marx, K., \& Engels, F. (1962) [1845]. Die heilige Familie oder Kritik der kritischen Kritik. In K. Marx \& F. Engels, Werke, Bd. 2 (S. 3-223). Berlin: Dietz.

Menzel, U. (2020). Der Corona-Schock. Die finale Entzauberung der Globalisierung. Blätter für deutsche und internationale Politik, 65(4), 37-44.

Stichweh, R. (2020). Simplifikation des Sozialen. Frankfurter Allgemeine Zeitung v. 07.04.2020, https:// zeitung.faz.net/faz/feuilleton/2020-04-07/simplifikation-des-sozialen/446149.html. Zugegriffen: Juni 2020. 